Neue Optionen für Lymphombehandlung

\section{Stabiles Konjugat-Therapeutikum}

\author{
Mit Brentuximab Vedotin wird in absehbarer Zeit eine neu- \\ artige konjugierte Substanz zur Verfügung stehen. Sie soll beim \\ Hodgkin-Lymphom (HL) und beim anaplastisch-großzelligen \\ Lymphom (ALCL) eingesetzt werden.
}

Das Antikörper-Drug-Konjugat (ADC) Brentuximab Vedotin besteht aus einem monoklonalen Antikörper gegen das Antigen CD30 und dem Zytostatikum Monomethyl-Auristatin. Beide Bestandteile sind über eine neuartige patentierte Linkertechnologie konjugiert und bleiben in der Blutbahn stabil miteinander verbunden. Nach der Bindung an das CD30-Antigen wird das
Konjugat in die Tumorzelle geschleust, der Linker durch lysosomale Enzyme gespalten und damit das Zytostatikum freigesetzt.

„Mit Brentuximab Vedotin steht erstmals seit 30 Jahren eine neue Therapieoption zur Verfügung, die auf einer zielgerichteten Elimination der malignen Zellpopulation beruht", erklärte Andreas Engert, Köln. Norbert Schmitz, Hamburg, ergänzte, dass die Be- handlung des therapierefraktären oder rezidivierenden ALCL neu bewertet werden müsse und dabei Brentuximab Vedotin eine zentrale Rolle zukommen könne. In Phase-II-Studien lagen die objektiven Ansprechraten auf Brentuximab Vedotin bei $75 \%$ (HL) bzw. $86 \%$ (ALCL). Eine vollständige Remission konnte bei $34 \%$ (HL) bzw. $57 \%$ (ALCL) erzielt werden.

Sowohl von der US-amerikanischen Zulassungsbehörde FDA als auch der europäischen EMA hatte das Konjugat bereits den Orphan-Drug-Status erhalten. Im August 2011 wurde Brentuximab Vedotin in den USA zugelassen. $\mathrm{AF}$

Nach Informationen von Takeda

\title{
Galenus-von-Pergamon-Preis 2012
}

\section{Ofatumumab - stark bei doppelt refraktärer CLL}

\begin{abstract}
Patienten mit chronischer lymphatischer Leukämie (CLL), die therapierefraktär gegenüber Fludarabin und Alemtuzumab sind, haben mit dem monoklonalen CD20-Antikörper Ofatumumab (Arzerra ${ }^{\oplus}$ ) von GlaxoSmithKline eine mediane Überlebenszeit von mehr als einem Jahr. Für diese Patienten gibt es also erstmals eine nachweislich wirksame und zugelassene Therapieoption.
\end{abstract}

Ein deutlicher Fortschritt für doppelt therapierefraktäre CLLPatienten ist der seit April 2010 EU-weit zugelassene monoklonale Anti-CD20-Antikörper Ofa-

\footnotetext{
Der Galenus-von- Pergamon-Preis würdigt in zwei Kategorien herausragende Arzneimittel-Innovationen und exzellente Forschungsleistung in der klinischen oder experimentellen Pharmakologie. Der Galenus-Preis ist in zwei Kategorien unterteilt:

- Kategorie A wird für eine hervorragende, in Deutschland bereits zugelassene Arzneimittel-Innovation vergeben.

- Kategorie B wird für eine hervorragende Forschungsleistung in der klinischen oder experimentellen Pharmakologie in Deutschland vergeben.

Der Galenus-von-Pergamon-Preis 2012 wird am 18. Oktober 2012 in Berlin verliehen. GlaxoSmithKline bewirbt sich mit Arzerra ${ }^{\oplus}$ in diesem Jahr für den Preis.
}

tumumab. In der zulassungsrelevanten internationalen Studie mit offenem, einarmigem Design hatten 95 CLL-Patienten, die auf eine Therapie mit Fludarabin und Alemtuzumab refraktär waren (doppelt refraktär), eine Monotherapie mit Ofatumumab erhalten. Die median 64 Jahre alten $\mathrm{Pa}$ tienten waren stark vorbehandelt, 59\% auch mit Rituximab. 59\% der Patienten befanden sich im BinetStadium C. Primärer Endpunkt der Studie war die objektive Ansprechrate, sekundäre Endpunkte Ansprechdauer, progressionsfreies Überleben, Gesamtüberleben und Therapiesicherheit.

$51 \%$ der Patienten sprachen auf die Monotherapie mit Ofatumumab an. Die mediane Gesamtüberlebenszeit betrug 14,2
Monate, das mediane progressionsfreie Überleben 5,5 Monate. Die Ansprechrate der Patienten, die bereits eine Rituximab-Vortherapie erhalten hatten, lagen mit $45 \%$ in ähnlicher Größenordnung wie beim Gesamtkollektiv. 18 Patienten waren FCR-refraktär. Selbst sie wiesen eine Ansprechrate von $44 \%$ auf. Bei 37 Patienten lag eine Deletion $17 p$ vor, bei ihnen betrug die Ansprechrate 37\%.

Die Behandlung mit Ofatumumab erwies sich im Allgemeinen als gut verträglich. Bei $63 \%$ der Patienten wurden initial Infusionsreaktionen beobachtet, die aber im Verlauf der weiteren Behandlung immer seltener auftraten. Sie waren in $95 \%$ der Fälle leicht bis mäßig ausgeprägt. $\mathrm{Zu}$ den häufigsten unerwünschten Ereignissen höheren Grades zählten Infektionen, Neutropenien und Anämien. Art und Schweregrad der Nebenwirkungen lagen aber für die stark vorbehandelten Patienten mit refraktärer CLL im erwarteten Rahmen.

mar 